\title{
Characterization of Hydraulic Power in Free-Stream Installations
}

\author{
Olivier Cleynen, Stefan Hoerner, and Dominique Thévenin \\ Lehrstuhl für Strömungsmechanik und Strömungstechnik (LSS), Otto-von-Guericke-Universität Magdeburg/ISUT,
Universitätsplatz 2, 39106 Magdeburg, Germany
}

Correspondence should be addressed to Olivier Cleynen; olivier.cleynen@ovgu.de

Received 5 January 2017; Revised 6 March 2017; Accepted 23 March 2017; Published 9 May 2017

Academic Editor: Ryoichi Samuel Amano

Copyright (C) 2017 Olivier Cleynen et al. This is an open access article distributed under the Creative Commons Attribution License, which permits unrestricted use, distribution, and reproduction in any medium, provided the original work is properly cited.

\begin{abstract}
The performance of open-channel hydropower devices can be optimized by maximizing the product of their load, hydraulic, and generator efficiencies. The maximum hydraulic power theoretically available must be defined according to the operational scenario retained for the device of interest. In the case of a device operating within a wide, unobstructed channel, the existence of a maximum hydraulic power and the operating speed required to reach it are first predicted using a one-dimensional flow model. This model is then extended to account for the effect of device ducting. As a result, given the available surface level drop and a single duct characteristic parameter, the model predicts the optimum device operating speed, whether the duct can improve performance, and the relative duct size which maximizes the installation's power density, all at a very low computational cost.
\end{abstract}

\section{Introduction}

It is now widely accepted that fluid flows in the environment represent a resource whose exploitation can make a significant contribution towards solving our current energy production challenge. While the bulk of this contribution currently comes from high-head hydraulic installations and axial wind turbines, research is also being carried out to optimize unconventional devices such as Wells [1], Savonius [2], or Darrieus [3] turbines, which are suited to specific installation sites or operating conditions.

One further area of interest is the exploitation of water currents with low-power, low-footprint devices [4]. Conventional micro-hydro $(<100 \mathrm{~kW})$ and pico-hydro $(<5 \mathrm{~kW})$ installations are associated with high global sustainability ratings [5]; in particular, devices operating without additional damming or modification to channel beds have an extremely low impact on fauna and flora and are most likely to meet the most stringent environmental regulations in Europe or even Germany. Because they feature relatively high availability and can be installed with relative ease near populated areas, machines such as floating water wheels and turbines may make a valuable contribution towards increasing electrification or decentralizing existing power networks.
It is immediately apparent, however, that these devices are afflicted with low-power densities and may feature relatively high power-specific acquisition costs. The first issue of maximizing efficiency is considered in the present publication, while the minimizing of associated costs is considered separately under the guidance of other project partners [6]. The complex nature of fluid flows within these machines (three-dimensional, turbulent, featuring free surfaces) makes it difficult to predict a priori their power potential. The final stages of the design of such turbomachines are in practice carried out using Computational Fluid Dynamics (CFD) simulations and experimental measurements. For such flows involving moving parts and free surfaces, considerable computational resources and time budgets are required. In addition, errors associated with the volume-of-fluid (VOF) technique, and especially the inherent uncertainties associated with the Reynolds averaging (RANS) of the momentum equations in CFD, require devoting additional resources to calibration and validation of simulation results.

In this view, simpler models permitting faster computations may provide an opportunity for early-stage optimization over a considerably broader range of parameters. Additionally, the interdependency of design and operating parameters-for example, the use of floats and rigs to duct 


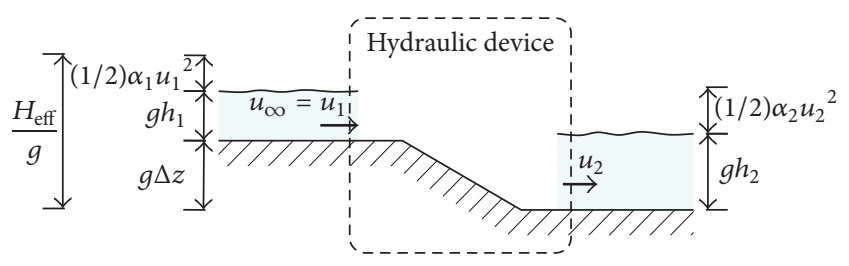

FIGURE 1: Notation for describing an arbitrary hydraulic installation, including the effective head $H_{\text {eff }}$ introduced by Pelz [7] and described in Section 3 of this paper.

water flow to the device affects both the power made available to the machine and its internal efficiency-is such that a theoretical analysis of the achievable performance is a first, essential step towards developing high-efficiency systems.

The motivation for this paper is thus to propose a framework to measure the efficiency of hydraulic devices operating in free-stream flows significantly larger than themselves. The parameters controlling the design and operation of such machines will be first derived. Optimal conditions will be identified. Then, the flow characteristics and performance changes resulting from the use of stationary ducts will be discussed.

\section{Parameters for Hydropower Performance Evaluation}

Several approaches can be taken for quantifying the performance of hydraulic power devices. The following criteria and notation, illustrated in Figure 1, are used in the present analysis.

Hydraulic power $\dot{W}_{\text {hydraulic }}$ is the time rate at which water affected by the machine is losing or gaining specific mechanical energy $e$ :

$$
\dot{W}_{\text {hydraulic }} \equiv \int(\Delta e) \mathrm{d} \dot{m}
$$

in which the integral is performed over the surface of a control volume enclosing the device, and in which the sign of $\Delta e$ is measured from the reference frame of the fluid (and thus usually negative - a loss).

In the case of a machine with uniform inlet and outlet properties, $\Delta e$ can be evaluated as follows:

$$
\Delta e=\Delta\left[g(z+h)+\frac{1}{2} u^{2}\right] .
$$

In that case and when the mass flow through the machine $\dot{m}$ is easily identified, hydraulic power (1) is simply evaluated as follows:

$$
\dot{W}_{\text {hydraulic }}=\dot{m} \Delta\left[g(z+h)+\frac{1}{2} u^{2}\right]
$$

Nevertheless, in the case of a device operating within a wide channel (such as a tidal turbine), it may be impossible to identify a single mass flow rate $\dot{m}$ corresponding to a streamtube with uniform properties, and $\dot{W}_{\text {hydraulic }}$ needs

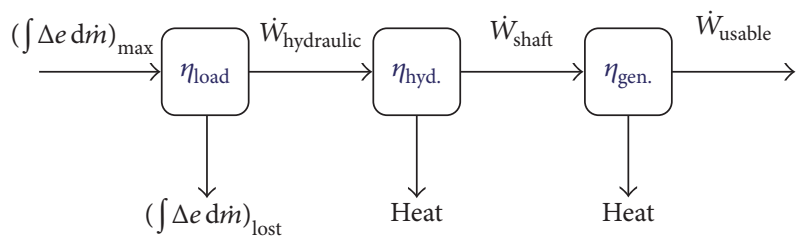

FIGURE 2: A conceptual representation of the energy flow through a machine operating in arbitrary conditions. The usable power $\dot{W}_{\text {usable }}$ is maximized when the three efficiencies $\eta_{\text {generator }}, \eta_{\text {hydraulic }}$, and $\eta_{\text {load }}$ are equal to one. The definition of what constitutes the maximum value of $\int \Delta e \mathrm{~d} \dot{m}$ depends on the operating constraints, as discussed later.

to be evaluated with integral (1), which requires extensive knowledge of the velocity field.

The hydraulic power transmitted to or from the device can have an extremum value for a given set of constraints. The existence and value of this maximum or minimum depend on the parameters which are assumed given, that is, on the chosen operational scenario. The purpose of the present document is to study and determine best design and operating conditions.

The energy flow through a hydraulic machine can be conceptualized as shown in Figure 2. The performance of a hydraulic machine is then evaluated using the following three efficiencies.

When there exists an extremum hydraulic power (see later discussion), then a device's ability to reduce the energy of the water is measured with the load efficiency $\eta_{\text {load }}$ :

$$
\eta_{\text {load }} \equiv \frac{\dot{W}_{\text {hydraulic }}}{\dot{W}_{\text {hydraulic max. }}} .
$$

The hydraulic power extracted from the water is in part converted to (useful) shaft power, while viscous effects cause the other part to be washed down the flow, ultimately translating into heat. A measure of the conversion effectiveness is already known as the hydraulic efficiency $\eta_{\text {hydraulic: }}$

$$
\eta_{\text {hydraulic }} \equiv \frac{\dot{W}_{\text {shaft }}}{\dot{W}_{\text {hydraulic }}} .
$$

Because of the high specific heat capacity of water, losses converted into heat do not translate into easily measurable temperature changes, in particular for free surface flows and for such low-power installations. In practice, a direct experimental measurement of the hydraulic efficiency of such an installation is therefore very difficult.

Lastly, the shaft power is converted into usable form, usually electricity, in a process whose effectiveness is measured with the generator efficiency $\eta_{\text {generator: }}$ :

$$
\eta_{\text {generator }} \equiv \frac{\dot{W}_{\text {usable }}}{\dot{W}_{\text {shaft }}} .
$$

Therefore, the usable power developed by a hydraulic installation can be expressed as follows:

$$
\dot{W}_{\text {usable }}=\eta_{\text {generator }} \eta_{\text {hydraulic }} \eta_{\text {load }} \dot{W}_{\text {hydraulic max. }} .
$$


Differing definitions for the efficiency may be constructed; for example, a different turbine efficiency may be devised to relate the shaft power to the flow wake impact [8]. Nevertheless, this study focuses on the energy conversion process. The three efficiencies in (7) are independent but may be affected together. For instance, the rotating speed of the impeller would impact both $\eta_{\text {generator }}$ and $\eta_{\text {hydraulic }}$. In what follows, the focus is on hydrodynamics and $\eta_{\text {generator }}$ is not further considered.

Hydraulic power can be nondimensionalized by comparing it to a partly arbitrary reference power: this ratio is named harvesting factor or power coefficient $C_{\mathrm{P}}$. A commonly accepted definition [9], used as well in this paper, is

$$
C_{\mathrm{P}} \equiv \frac{\dot{W}_{\text {hydraulic }}}{(1 / 2) \rho A_{\mathrm{f}} u_{\infty}^{3}} .
$$

Other selections of reference powers for the denominator of (8) can be used, such as those proposed by Li [10], Denny [11], or that put forth by Pelz [7], that is discussed below and rewritten here with our notation:

$$
C_{\mathrm{P}} \equiv \frac{-\dot{W}_{\text {hydraulic }}}{(4 / 5) \dot{m}\left(g h_{1}+\left(\alpha_{1} / 2\right) u_{1}^{2}-g \Delta z\right)} .
$$

At that point, it is important to stress out that the efficiency values $\eta$ introduced previously have an unambiguous definition and fulfill the condition $\eta \leq 1$. On the other hand, the power coefficient is merely a nondimensional parameter with a partly arbitrary character. As a result, the value of $C_{\mathrm{P}}$ might even exceed 1 . Different values for the maximum power coefficient $C_{\mathrm{P} \text { max. }}$ may be attributed to a given machine, because either the underlying assumption in defining the optimum case or the definition of $C_{\mathrm{P}}$ may differ. These are the only reasons why Pelz's limit described later in (11) does not converge towards Betz's classical result when $g \rightarrow 0 \mathrm{~m} \mathrm{~s}^{-2}$ and $\Delta z \rightarrow 0 \mathrm{~m}$.

Given this context, and considering only steady fluid flows with uniform inlet and outlet properties, the open question raised in this paper is "which optimum operating conditions will maximize the hydraulic power of any arbitrary device subjected to given altitude drop and viscous losses?"

\section{Maximum Power in Obstructed Channel Flow}

In order to connect our new developments with established results from the literature, the analysis starts by considering an obstructed channel. Pelz first developed (2011, [7]) an expression for an extremum hydraulic power $\dot{W}_{\text {hydraulic max }}$ with the following constraints:

(1) A full obstruction of the upstream fluid flow

(2) An entirely unobstructed downstream flow

(3) A limited outlet width.

Thus, in this model, condition (2) turns the outlet water height $h_{2}$ into a control variable, while condition (1) sets the mass flow $\dot{m}$, and condition (3) sets the outlet width $b_{2}$, as input constants. The known mass flow $\dot{m}$ passes through the device at all times.

To analyze the system, Pelz defines an energy budget termed effective head $H_{\text {eff. }} \equiv h_{1}+\alpha_{1}\left(u_{1}^{2} / 2 g\right)-\Delta z$, using the notations shown in Figure 1 and in Nomenclature. Pelz then shows that the hydraulic power available to an installation fed with rectangular ducts can be maximized if two conditions are met. They constrain the Froude number at exit $F_{2} \equiv$ $u_{2, \text { av. }} / \sqrt{g h_{2}}$ and the volume flow per unit width at exit $q_{2} \equiv$ $\dot{\mathscr{V}}_{2} / b_{2}=u_{2, \text { av. }} h_{2} / b_{2}$, leading to following conditions:

$$
\begin{aligned}
& q_{2, \text { opt. }}=\left(\frac{g}{\alpha_{2}}\right)^{1 / 2}\left[\frac{2}{5} H_{\text {eff. }}\right]^{3 / 2}, \\
& F_{2, \text { opt. }}=1 .
\end{aligned}
$$

The maximum hydraulic power that may be extracted from a flow of given $H_{\text {eff. }}$ when $\eta_{\text {hydraulic }}=1$ is then written in our notation:

$$
\dot{W}_{\text {hydraulic max,Pelz }}=-\rho b_{2}\left(\frac{g^{3}}{\alpha_{2}}\right)^{1 / 2}\left[\frac{2}{5} H_{\text {eff. }}\right]^{5 / 2} .
$$

This optimum, which can be rewritten as $\dot{W}_{\text {hydraulic max,Pelz }}=$ $-\dot{m} g(2 / 5) H_{\text {eff. }}$, is a benchmark to evaluate the performance of hydraulic machines working with a fully controlled stream, that is, in installations where the fluid is guaranteed to enter the device and where the outlet flow can be discharged with any chosen water level height $h_{2}$. Indeed, during performance evaluations of such devices, the mass flow rate $\dot{m}$ is a direct input variable [12].

The removal of condition (1) (full flow obstruction, with the entire stream passing through the device) has been later explored by Pelz and Metzler [13]: a model is then obtained for the performance of channel hydraulic devices installed on the bottom of channels and with lateral flow bypass.

Even if these theoretical findings have a pioneering character and remain of great use, many hydraulic devices exist for which conditions (2) and (3), necessary for the derivation of the above optimum, do not apply. Such devices may include floating installations operating in wide and/or deep channels, for example, operated where the tidal power potential is highest [14] or away from navigation lanes. In that case,

(i) the mass flow $\dot{m}$ flowing through the device is not readily known because it is a function of the operating conditions;

(ii) it cannot be assumed that an unobstructed, steeply sloped bed at altitude $z_{2}$ is available at the outlet to receive the water leaving the machine. Instead, the outlet boundary condition will be conditioned by the water level altitude $\left\{z_{2}+h_{2}\right\}$ of an existing body of water (e.g., a reservoir) independent of the device operation.

A machine operating in such conditions would therefore attain a maximum hydraulic power different from that quantified in (11). For such a case, constraining the value of $\dot{m}$ as 


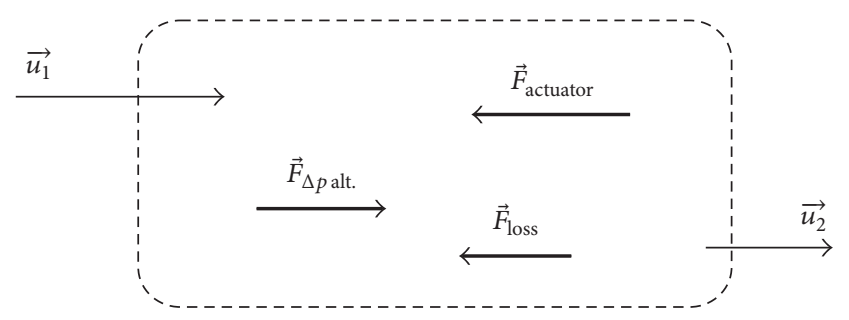

FIGURE 3: A hydraulic machine in the most generic case: the fluid momentum is altered by the combined action of forces $\vec{F}_{\text {actuator }}$, $\vec{F}_{\Delta p_{\text {alt. }}}$, and $\vec{F}_{\text {loss }}$.

input variable, as in [15], would result in grossly overestimated power calculations. The purpose of the next section is to determine a realistic prediction of optimal operation.

\section{Maximum Power in Unobstructed Channel Flow}

A model is developed here to evaluate the performance of a hydraulic power device operating in a wide channel and constrained by a given outlet water altitude $\left\{z_{2}+h_{2}\right\}$. This model is based on the classical actuator disk models developed by Froude, Betz, Joukowski, and other scientists (described, e.g., by van Kuik et al. in 2015 [16]). Building on top of this classical theory, the effects of altitude change and duct pressure loss will be included in the analysis. This model assumes uniform inlet and outlet velocities; however, advances made in the modeling of wakes [17] and wall effects [18] may lift this restriction in the future.

In the most general case, a hydraulic machine can be described as shown in Figure 3; water incoming at 1 and leaving at 2 sees its momentum changed by the three forces $\vec{F}_{\text {actuator }}$ (by the power-producing moving parts), $\vec{F}_{\Delta p_{\text {alt. }}}$ (due to altitude change), and $\vec{F}_{\text {loss }}$ (due to internal friction effects):

$$
\vec{F}_{\text {actuator }}+\vec{F}_{\Delta p_{\text {alt. }}}+\vec{F}_{\text {loss }}=\dot{m}\left(\vec{u}_{2}-\vec{u}_{1}\right) \text {. }
$$

When one considers the machine as a black box, the hydraulic power production can be described as a one-dimensional phenomenon. Here, for clarity, the machine is arranged so that all forces and velocities are aligned with the horizontal direction, $x$.

In an ideal machine designed for this environment, water would be guided to and from an actuator surface, across which hydraulic power is extracted from the fluid (Figure 4). The velocity changes of the fluid are matched with crosssectional area changes along the flow, so that the pressure distribution within the device can be traced as in Figure 5 and quantified as follows.

The force exerted by the ideal actuator can be expressed in two different ways, one as a function of the rate of change of momentum of the water:

$$
\begin{aligned}
F_{\text {actuator }} & =\dot{m}\left(u_{2}-u_{1}\right)-F_{\Delta p_{\text {alt. }}}-F_{\text {loss }} \\
& =\rho u_{\mathrm{A}} A_{\mathrm{A}}\left(u_{2}-u_{1}\right)+\rho g A_{\mathrm{A}} \Delta(z+h)-F_{\text {loss }}
\end{aligned}
$$

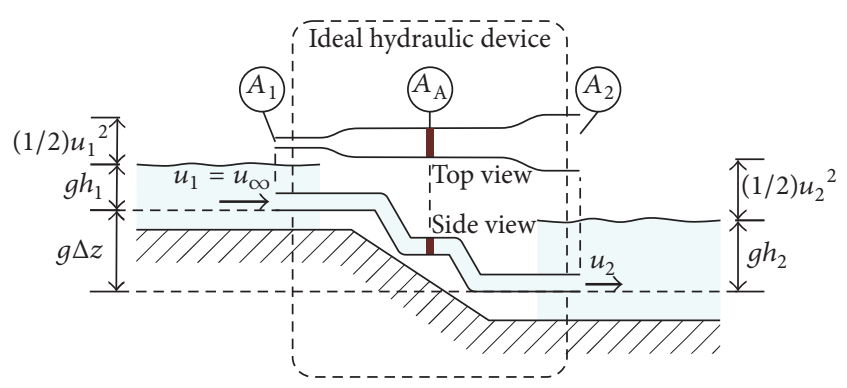

FIGURE 4: Cross section of an arbitrary ideal hydraulic installation. The water streamtube expands from cross-sectional area $A_{1}$ to $A_{\mathrm{A}}$, with a corresponding increase in pressure. The actuator surface of area $A_{\mathrm{A}}$ extracts hydraulic power from the fluid with a resulting pressure drop; the streamtube then expands again so the outlet pressure reaches $p_{2}$. The altitudes of the inlet, outlet, and actuator surface do not affect the machine's performance and are never specified. In this model, neither $h_{1}$ nor $h_{2}$ are affected by the power extracted by the device, since it occupies only a small part of the channel.

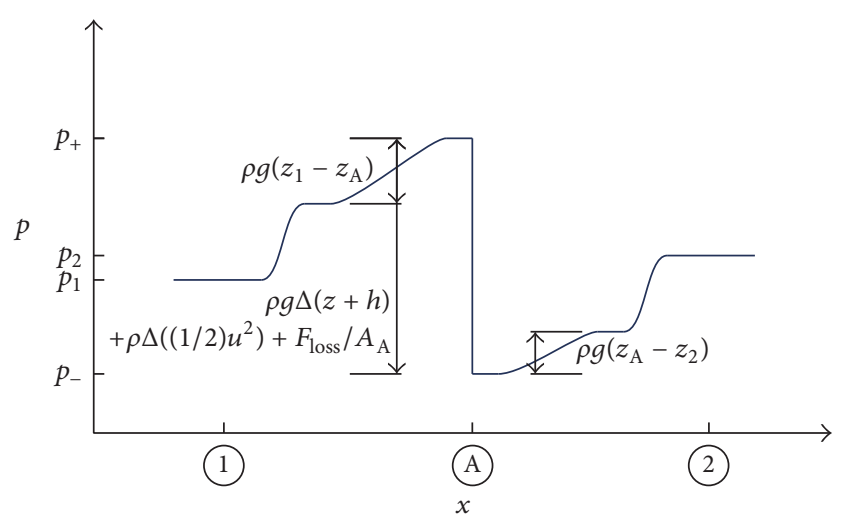

FIGURE 5: Pressure distribution within the arbitrary ideal machine described in Figure 4. The pressure $p_{1}$ of the inlet water is raised twice: first as its velocity is reduced from $u_{1}$ to $u_{\mathrm{A}}$ and then as the altitude is varied by $z_{1}-z_{\mathrm{A}}$. Similarly, downstream of the actuator, the fluid pressure is raised twice so that flow conditions (2) at the outlet may be attained. The pressure drop across the actuator surface A, described in (14), corresponds to the lumped contributions of net water level altitude change $\Delta(z+h)$, velocity change $(1 / 2) \Delta u^{2}$, and losses due to drag $F_{\text {loss }}$.

and the other as a function of the kinetic energy change across the actuator surface:

$$
\begin{aligned}
F_{\text {actuator }} & =A_{\mathrm{A}}\left(p_{+}-p_{-}\right) \\
& =\rho A_{\mathrm{A}}\left[\frac{1}{2}\left(u_{2}^{2}-u_{1}^{2}\right)+g \Delta(z+h)\right]-F_{\text {loss }} .
\end{aligned}
$$

In this analysis, the sign convention is as described in Figure 3; that is, $\Delta(z+h)<0$ when the water level drops and $F_{\text {loss }}$ is always negative; thus, whenever hydraulic power is produced by the device, $F_{\text {actuator }}<0$. 
Equating (13) and (14) gives an expression of $u_{\mathrm{A}}$ as a function of $u_{1}$ and $u_{2}$; this allows to express the hydraulic power as

$$
\begin{aligned}
& \dot{W}_{\text {hydraulic }}=\rho A_{\mathrm{A}} \frac{1}{2}\left(u_{2}+u_{1}\right) \\
& \cdot\left[\frac{1}{2}\left(u_{2}^{2}-u_{1}^{2}\right)+g \Delta(z+h)-\frac{F_{\text {loss }}}{\rho A_{\mathrm{A}}}\right], \\
& \dot{W}_{\text {hydraulic }} \\
& \quad=\rho A_{\mathrm{A}} u_{\mathrm{A}}\left[2 u_{\mathrm{A}}^{2}-2 u_{\mathrm{A}} u_{1}+g \Delta(z+h)-\frac{F_{\text {loss }}}{\rho A_{\mathrm{A}}}\right] .
\end{aligned}
$$

This expression reduces as expected to that of Betz in the case where either $\Delta(z+h)$ or $g$ tend to zero. Similarly, when $u_{1}=u_{2}$, that is, no kinetic energy is withdrawn from water, the power tends towards $\dot{W}_{\text {hyd. }}=\rho A_{\mathrm{A}} u_{1} g \Delta(z+h)$, as expected of a high-head hydropower installation.

In order to maximize power, a compromise must be made at the actuator surface: higher actuator velocities increase the mass flow but reduce kinetic energy harvest; while lower actuator velocities increase kinetic energy recovery at the cost of decreased mass flow. This compromise is additionally affected by the (usually negative) potential energy term $g \Delta(z+h)$ and the losses $F_{\text {loss }}$ due to friction.

In this paper, the optimum is determined according to following constraints:

\section{(1) A known device actuator surface area}

(2) A known water surface altitude drop independent of the device

Here, condition (1) constrains the actuator surface area $A_{\mathrm{A}}$, while condition (2) constrains the water level drop $\Delta(z+$ $h)$ as input variables; therefore, the main control variable becomes the cross-actuator velocity $u_{\mathrm{A}}$. Alternative boundary conditions describing, for example, a bypass flow [19] may be added in the future.

In the view of these restrictions, (15b) is nondimensionalized by using the power coefficient defined in (8) and the ratio $u_{\mathrm{A}} / u_{\infty}$, which is equal to $u_{\mathrm{A}} / u_{1}$ in our case. Additionally, a nondimensional drop coefficient $K_{\mathrm{D}}$ is defined to express the effect of both the water level drop and the friction losses, lumped together:

$$
K_{\mathrm{D}} \equiv-\frac{\rho g \Delta(z+h)-F_{\text {loss }} / A_{\mathrm{A}}}{(1 / 2) \rho u_{\infty}^{2}} .
$$

In this manner, the hydraulic power equation is obtained as follows:

$$
C_{\mathrm{P}}=4\left(\frac{u_{\mathrm{A}}}{u_{\infty}}\right)^{3}-4\left(\frac{u_{\mathrm{A}}}{u_{\infty}}\right)^{2}-K_{\mathrm{D}}\left(\frac{u_{\mathrm{A}}}{u_{\infty}}\right) .
$$

The solution of (17) is plotted in Figure 6, in which it is seen that unfavorable (negative) values of $K_{\mathrm{D}}$ decrease not only the maximum hydraulic power attainable by the machine (this was obviously expected) but also the optimum actuator speed needed to attain it. For $K_{\mathrm{D}}<-1$, the power

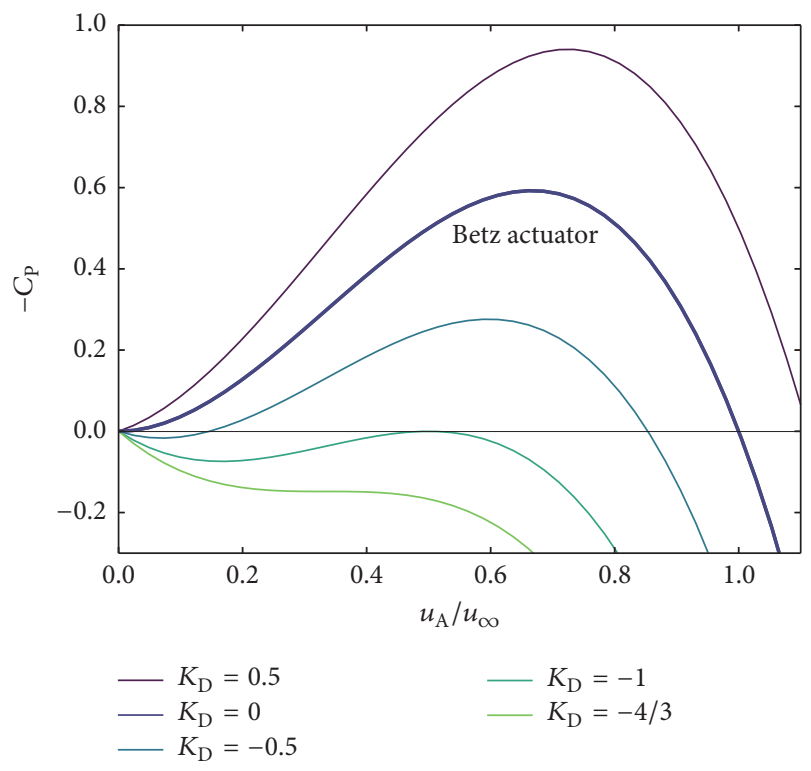

Figure 6: Power curves for an ideal actuator surface machine, for different values of the drop coefficient $K_{\mathrm{D}}$. The case $K_{\mathrm{D}}=0$ corresponds to the well-known solution for an actuator surface without any loss, now associated with Betz and Joukowski. Positive values of $K_{\mathrm{D}}$ correspond to increased pressure drop across the device; increasingly negative values of $K_{\mathrm{D}}$ may occur due to increasing pressure losses. Note that negative $C_{\mathrm{P}}$ values (or $-C_{\mathrm{P}}>0$, above the straight horizontal line in this figure) correspond to hydraulic power being extracted by the device, which is obviously the objective.

coefficient is always positive (the device turns into a pump and does not extract any energy from the fluid); for $K_{\mathrm{D}}<$ $-4 / 3$, the power curve no longer features an extremum outside of the standing condition $\left(u_{\mathrm{A}}=0\right)$.

The value of $u_{\mathrm{A}} / u_{\infty}$ that produces extremum values for the hydraulic power (i.e., setting $\eta_{\text {load }}=1$ ), as well as an explicit expression for this extremum, can be found by differentiating (17) with respect to $u_{\mathrm{A}} / u_{\infty}$ and selecting the roots relevant to this study. For clarity, those are expressed as a function of a factor $T \equiv(1 / 3)\left[1+(3 / 4) K_{\mathrm{D}}\right]^{1 / 2}$, obtaining

$$
\begin{aligned}
\frac{u_{\text {A opt. }}}{u_{\infty}} & =\frac{1}{3}+T, \\
C_{\text {P extremum }} & =-\frac{8}{27}+4 T^{3}-\frac{4}{3} T-\left(\frac{1}{3}+T\right) K_{\mathrm{D}} .
\end{aligned}
$$

In the case where there is neither altitude drop nor friction loss, the drop coefficient $K_{\mathrm{D}}$ is brought to zero and $T \rightarrow 1 / 3$ : then, $u_{\text {A opt. }} \rightarrow(2 / 3) u_{\infty}$; and the absolute value of the power coefficient tends towards $16 / 27$, a classical value known as the Betz-Joukowski limit [16]. (It may be worth noting that Bergey [20] and van Kuik in 2007 [21] propose attributing this result to Lanchester as well. Van Kuik et al. in 2015 [16], however, show that although he expanded Froude's work to reach the result $\left|C_{\mathrm{P} \text { extremum }}\right|=16 /\left(27 \mathrm{Q}^{2}\right)$, Lanchester failed to conclude definitely on the value of the factor $Q$, which shifts the value of the optimum velocity ratio due to an imbalance which he introduced in the actuator model [22]. 


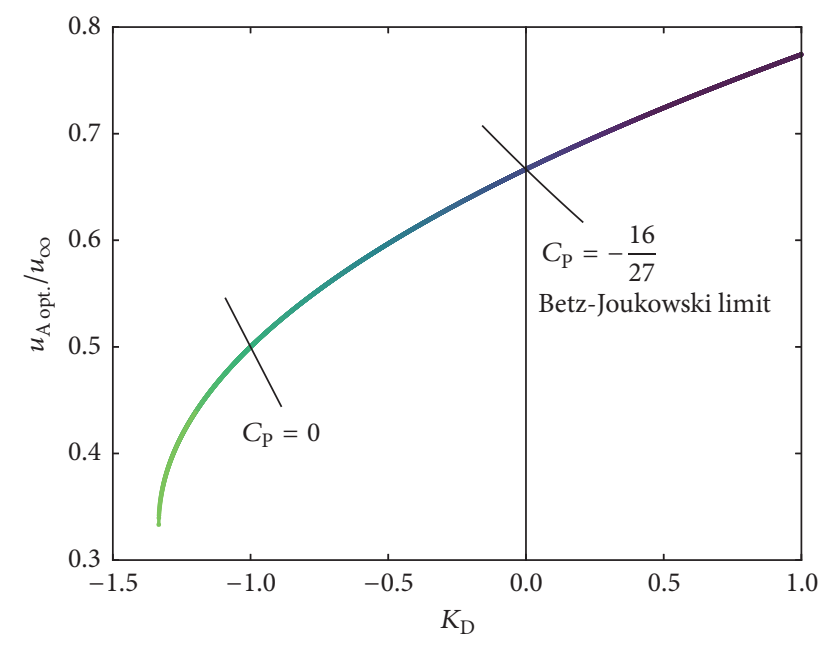

FIgURE 7: Optimum nondimensional actuator velocity $u_{\text {A opt. }} / u_{\infty} \equiv$ $u_{\mathrm{A}} /\left.u_{\infty}\right|_{\eta_{\text {load }}=1}$ as a function of the drop coefficient $K_{\mathrm{D}}$. The curve colormap is matched to that of Figure 6. For values below -1 , the power coefficient becomes positive, and no energy can be extracted; below $-4 / 3$, an optimum actuator velocity no longer exists, since the best efficiency is obtained for standing conditions.

For historical interest only, the value of $Q$, including some complex values, may be expressed as a function of $K_{\mathrm{D}}$ by equating (19) to $-16 / 27 Q^{2}$.)

The relationship between the optimum actuator velocity and the drop coefficient value (see (18)) is plotted in Figure 7. An optimum only exists for $K_{\mathrm{D}}>-4 / 3$, since standing conditions are found to be best when $K_{\mathrm{D}} \leq-4 / 3$. The machine can only extract hydraulic power when $K_{\mathrm{D}}>-1$. The optimum value $u_{\mathrm{A} \text { opt. }}$ increases monotonically with $K_{\mathrm{D}}$ : more favorable drop coefficients always shift the optimum velocity ratio upwards. This remains true even in the hypothetical case where $u_{\text {A opt. }}>u_{\infty}$, as the energy expenditure required to accelerate the flow through the device (in (15a), $\dot{m}\left(u_{2}^{2}-\right.$ $\left.u_{1}^{2}\right)>0$ ) is compensated by the resulting increase in mass flow. In a free-stream unchanneled installation (e.g., a floating or bed-bound tidal or river turbine), it is expected that $\Delta(z+h)=0$ and such devices will therefore always operate at $-1<K_{\mathrm{D}} \leq 0$.

\section{Performance Improvements through Ducting}

The developed model can now be expanded to account for the effect of ducting around a free-stream device. A suitable stationary duct positioned around a power-producing device can result in three distinct benefits:

(1) An increase in $\eta_{\text {hydraulic }} \eta_{\text {generator }}$, through the optimization of the flow velocity distribution or rotation speed

(2) An increase in power density obtained through the reduction in the size of the moving parts for a given volume footprint of the device
(3) An increase of power obtained through the increase of the frontal area caused by the presence of the duct

The first of those cannot be described in general terms for a generic hydraulic machine and is best studied with the help of experimental and CFD techniques. The last two, however, can be examined by extending the model described above, in order to obtain tentative quantitative descriptions based on sensible hypotheses, and especially a broad qualitative characterization of the phenomenon.

A duct may increase the flow velocity locally but will always impede the overall flow through and around the device; thus, in order to determine the optimal duct size (that which will maximize power or power density), its effectiveness must be coupled back with the drop coefficient $K_{\mathrm{D}}$. Two possibilities are explored in this paper.

5.1. Simple Duct Loss Behavior. A very simple duct drag model, in the line of classical hydrodynamic theory, links net drag force $F_{\text {loss }}$ to area $A_{\mathrm{f}}$ and to the square of the actuator flow velocity $u_{\mathrm{A}}$. The proportionality constant is termed $d \mathrm{rag}$ coefficient $K_{\mathrm{D} 2}$ :

$$
K_{\mathrm{D} 2} \equiv \frac{-F_{\text {loss }}}{(1 / 2) \rho u_{\mathrm{A}}^{2} A_{\mathrm{f}}} .
$$

In turn, the effect of altitude drop is described with a static drop coefficient $K_{\mathrm{D} 0}$ :

$$
K_{\mathrm{D} 0} \equiv \frac{-\rho g \Delta(z+h)}{(1 / 2) \rho u_{\infty}^{2}} .
$$

It is expected that, for a device of known geometry, neither $K_{\mathrm{D} 0}$ nor $K_{\mathrm{D} 2}$ are significantly affected by the device scale, the actuator speed, or the hydraulic power.

The drop coefficient $K_{\mathrm{D}}$ of (16) can now be rewritten as follows:

$$
K_{\mathrm{D}}=K_{\mathrm{D} 0}-K_{\mathrm{D} 2} \frac{A_{\mathrm{f}}}{A_{\mathrm{A}}}\left(\frac{u_{\mathrm{A}}}{u_{\infty}}\right)^{2} .
$$

The actuator speed ratio that maximizes the load efficiency, $u_{\mathrm{A} \text { opt. }} / u_{\infty}$, can now be described as a function of $K_{\mathrm{D} 0}$ and $K_{\mathrm{D} 2}$. The case where $A_{\mathrm{f}}=A_{\mathrm{A}}$ (corresponding to the central case, $R=1$, in Figure 9) is presented in Figure 8, where it is seen, as expected, that increases in the value of the drag coefficient lead to a decrease in the optimum velocity ratio and inevitably reduce the power output.

In a final effort to describe more realistic configurations, the geometry of the duct is parametrized using a size ratio parameter $R$ comparing the frontal area $A_{\mathrm{f}}$ of the device to that of the actuator, $A_{\mathrm{A}}$ (see also Figure 9):

$$
R \equiv \frac{A_{\mathrm{A}}}{A_{\mathrm{f}}}
$$

Then, (17) can be reformulated using (22) and (23) as follows:

$$
\begin{aligned}
C_{\mathrm{P}}= & {\left[4+\frac{K_{\mathrm{D} 2}}{R^{2}}\right]\left(R \frac{u_{\mathrm{A}}}{u_{\infty}}\right)^{3}-4\left(R \frac{u_{\mathrm{A}}}{u_{\infty}}\right)^{2} } \\
& -K_{\mathrm{D} 0}\left(R \frac{u_{\mathrm{A}}}{u_{\infty}}\right) .
\end{aligned}
$$




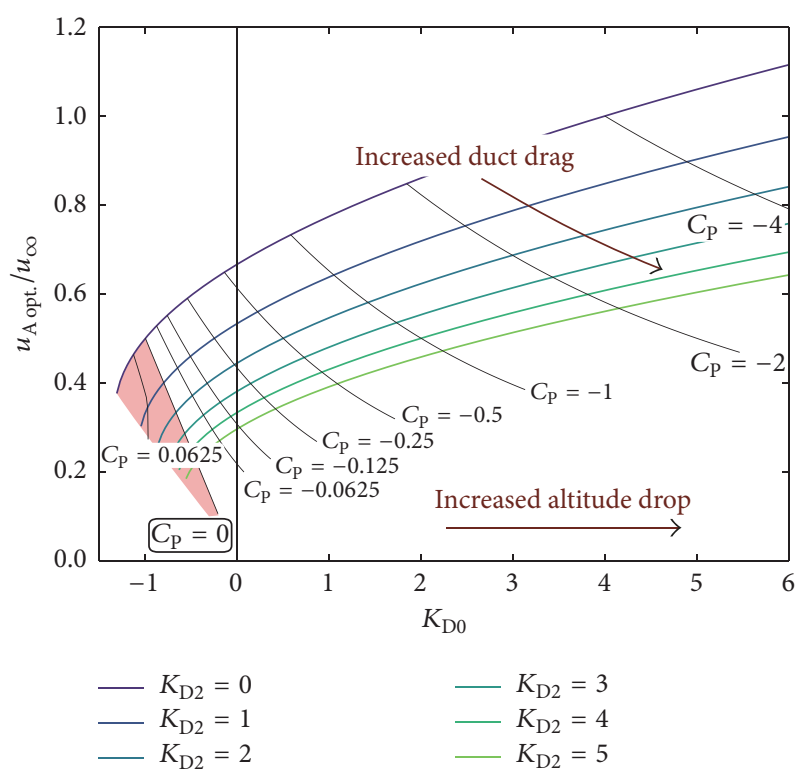

FIGURE 8: The optimum velocity ratio $u_{\mathrm{A} \text { opt. }} / u_{\infty} \equiv u_{\mathrm{A}} /\left.u_{\infty}\right|_{\eta_{\text {load }}=1}$ as a function of the static drop coefficient $K_{\mathrm{D} 0}$ and drag coefficient $K_{\mathrm{D} 2}$ in a device for which $A_{\mathrm{f}}=A_{\mathrm{A}}$. Isocurves for various values of $C_{\mathrm{P}}$ are also shown; the area highlighted in red, where the hydraulic power is positive (i.e., received by the water) is merely of theoretical interest. This diagram presents the solutions to (24) in which (25) is inserted, when $R=1$.

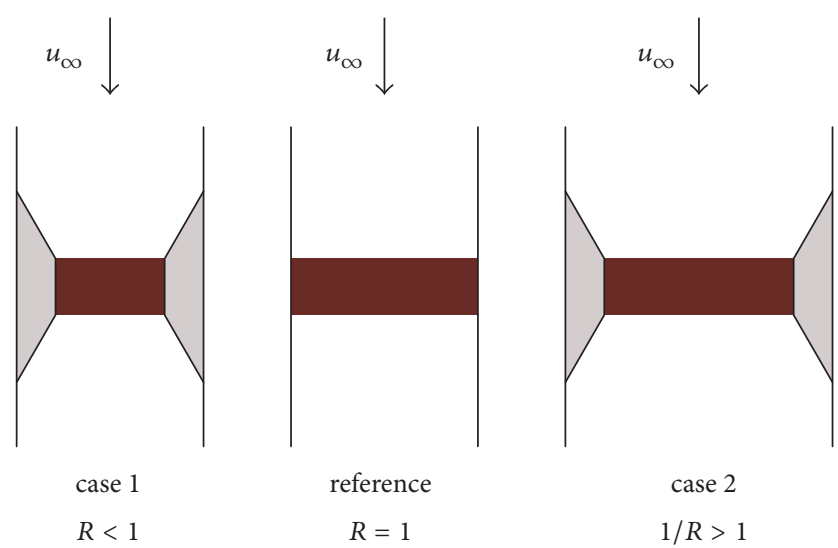

Figure 9: Conceptual schematic to describe the ducting of an actuator device. A duct can be used either to reduce the size of the actuator for a given frontal surface (case 1, left) or to increase the frontal area of a machine with an existing actuator (case 2, right). Both processes are described mathematically by reducing the value of parameter $R$ below 1 .

Since only the factor $\left(R\left(u_{\mathrm{A}} / u_{\infty}\right)\right)$ appears in (24) when the drag coefficient $K_{\mathrm{D} 2}$ is zero, any increase in $u_{\mathrm{A}}$ generated by ducting is exactly compensated by a decrease in the size ratio $R$, so that the power coefficient is unaffected. In practice, however, $K_{\mathrm{D} 2}>0$ and losses to friction are a function not merely of the free-stream velocity $u_{\infty}$ but also of the actuator operating velocity $u_{\mathrm{A}}$.
Expression (24) is maximized, yielding $\eta_{\text {load }}=1$, when the adjusted actuator velocity ratio $R\left(u_{\mathrm{A}} / u_{\infty}\right)$ reaches the following value:

$$
\begin{aligned}
& \left(R \frac{u_{\mathrm{A}}}{u_{\infty}}\right)_{\text {opt. }} \\
& =\frac{4+\left[16+12 K_{\mathrm{D} 0}+3 K_{\mathrm{D} 0}\left(K_{\mathrm{D} 2} / R^{2}\right)\right]^{1 / 2}}{12+3\left(K_{\mathrm{D} 2} / R^{2}\right)} .
\end{aligned}
$$

This optimum reduces to that of (18) when $K_{\mathrm{D} 2}=0$ and to the value $2 / 3$ predicted by Betz when both $K_{\mathrm{D} 0}=0$ and $K_{\mathrm{D} 2}=0$. Inserting (25) into (24) gives us an expression for the optimum power coefficient $C_{\mathrm{P} \text { opt. }}$ as a function of the size ratio for any given duct.

We are now able to characterize and visualize the effect of ducting on free-stream hydropower installations. This is perhaps best done by differentiating between two cases, illustrated in Figure 9. Compared to the reference configuration (a duct without any change in cross section) shown in the middle $(R=1)$,

(i) in case 1 , a duct with known characteristic drag coefficient $K_{\mathrm{D} 2}$ is used in the design phase to reduce the actuator size $A_{\mathrm{A}}$ for a given inlet frontal area $A_{\mathrm{f}}$. In this case, $R$ is reduced with the intent of increasing the power density $(1 / R) C_{\mathrm{P}}$;

(ii) in case 2 , a duct with known $K_{\mathrm{D} 2}$ is added on an existing device with fixed actuator area $A_{\mathrm{A}}$. In that case, $1 / R$ is increased with the intent of increasing the overall power $\dot{W}_{\text {hydraulic }}$.

The effect of both modifications, which amount to the same physical effect, can be observed in Figures 10 and 11, which represent how the power coefficient and power density evolve as $R$ is varied, for various values of $K_{\mathrm{D} 2}$.

It is readily seen from those figures that low drag coefficients are associated with a high increase in power density through the use of ducting; the ratio $R$ (size of actuator relative to frontal surface) then features an optimum value plotted in Figure 11. Nevertheless, high $K_{\mathrm{D} 2}$ values result in cases where adding a duct results in neither power nor power density increase (i.e., $R_{\text {opt. }}=1$ ) in that configuration. As expected, unless the frontal area is increased, the use of ducting can only result in a power coefficient decrease.

The geometrical features of the power and power density curves displayed in Figures 10 and 11 are also affected by the value of the drop coefficient $K_{\mathrm{D} 0}$. This dependence can be visualized in Figure 12, in which the value $R_{\mathrm{opt}}$ of the size ratio which maximizes the power density $(1 / R) C_{\mathrm{P} \text { max. }} / C_{\mathrm{P} \text { ref. }}$ is plotted as a function of $K_{\mathrm{D} 0}$ and $K_{\mathrm{D} 2}$.

From Figure 12, it is seen that, for any one value of $K_{\mathrm{D} 0}$ (a property of the operating environment), increasing values of the drag coefficient increase the optimum size ratio, that is, the relative size of the actuator that will maximize power density. For each value of $K_{\mathrm{D} 0}$, there exists a drag coefficient value above which $R_{\text {opt. }}=1$ : in that case, the ducting can only reduce performance. 


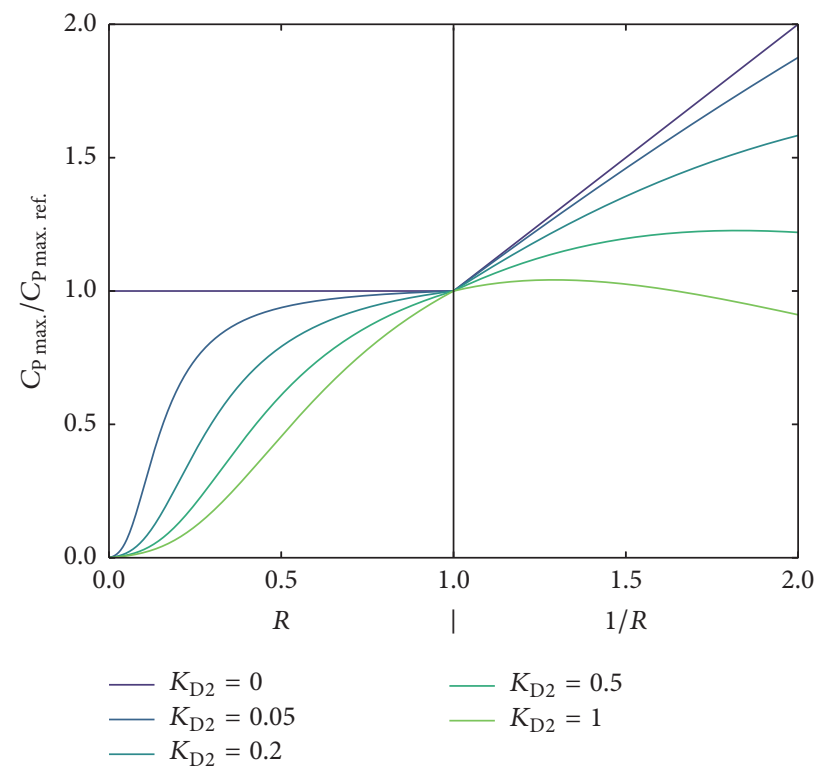

Figure 10: The ratio of maximum power coefficient $C_{\mathrm{P} \max }$ to a reference value $C_{\mathrm{P} \text { max.ref. }}=\left.C_{\mathrm{P} \max .}\right|_{R=1}$, shown as a function of the duct size ratio. The abscissa represents $R$ from values 0 to 1 and $1 / R$ from 1 onwards. The curves are drawn for various values of $K_{\mathrm{D} 2}$ when $K_{\mathrm{D} 0}=0.25$.

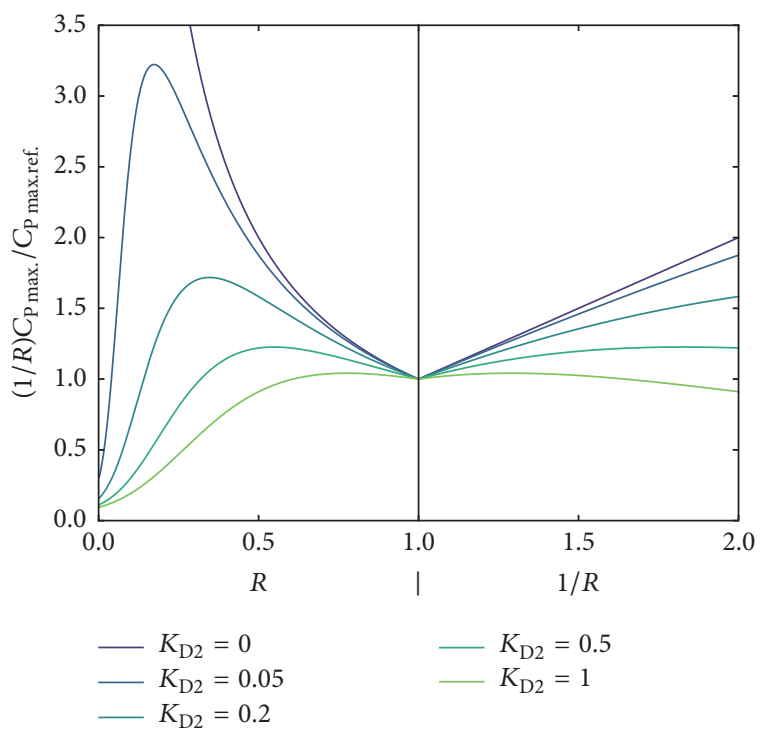

FIGURE 11: The ratio of maximum power density $(1 / R) C_{\mathrm{P} \text { max. }}$ to its reference value $\left.(1 / R) C_{\mathrm{P} \text { max. }}\right|_{R=1}$, shown as a function of the duct size ratio. The abscissa represents $R$ from values 0 to 1 and $1 / R$ from 1 onwards. The curves are drawn for various values of $K_{\mathrm{D} 2}$ when $K_{\mathrm{D} 0}=$ 0.25 .

5.2. Two-Component Drag Model. The methodology presented above can be used with differing drag models, suitable for describing more complex dependencies on the flow conditions. Such models may be constructed based on data obtained from specific experimental or CFD investigations. A generic example is proposed as follows for illustration. The drag generated by the duct could have two components:

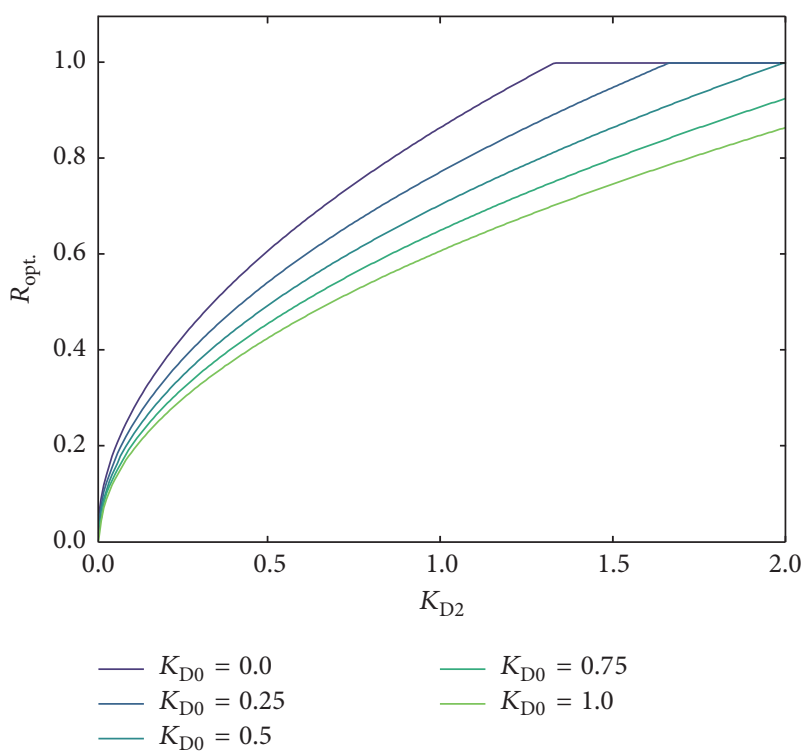

FIgURE 12: The optimum actuator size ratio $R_{\text {opt. }}$ (that will result in maximum hydraulic power density $\left.(1 / R) C_{\mathrm{P} \text { max. }} / C_{\mathrm{P} \text { ref. }}\right)$ plotted as a function of the drag coefficient $K_{\mathrm{D} 2}$ for various values of the static drop coefficient $K_{\mathrm{D} 0}$.

one proportional to the square of the actuator flow velocity, and the other proportional to the square of the free-stream velocity. Proportionality to the duct wet surface (rather than to the device frontal area) could also be made part of the model. Following those hypotheses, the drag would come up as a function of an inner and an outer drag coefficient $K_{\mathrm{D} 3}$ and $K_{\mathrm{D} 4}$ :

$$
-F_{\text {loss }}=\frac{1}{2} \rho(1-R) A_{\mathrm{f}}\left(K_{\mathrm{D} 3} u_{\mathrm{A}}^{2}+K_{\mathrm{D} 4} u_{\mathrm{\infty}}^{2}\right) .
$$

This would lead us to rewrite the drop coefficient and power coefficients from (16) and (24) as follows:

$$
\begin{aligned}
K_{\mathrm{D}}= & K_{\mathrm{D} 0}-K_{\mathrm{D} 3} \frac{1-R}{R}\left(\frac{u_{\mathrm{A}}}{u_{\infty}}\right)^{2}-K_{\mathrm{D} 4} \frac{1-R}{R} \\
C_{\mathrm{P}}= & {\left[4+K_{\mathrm{D} 3} \frac{1-R}{R^{3}}\right]\left(R \frac{u_{\mathrm{A}}}{u_{\infty}}\right)^{3}-4\left(R \frac{u_{\mathrm{A}}}{u_{\infty}}\right)^{2} } \\
& -\left[K_{\mathrm{D} 0}-K_{\mathrm{D} 4} \frac{1-R}{R}\right]\left(R \frac{u_{\mathrm{A}}}{u_{\infty}}\right)
\end{aligned}
$$

and the adjusted actuator velocity ratio $R\left(u_{\mathrm{A}} / u_{\infty}\right)$ for which the power coefficient that is at an extremum would then be derived as a cumbersome but still easily obtained expression:

$$
\begin{aligned}
& \left(R \frac{u_{\mathrm{A}}}{u_{\mathrm{\infty}}}\right)_{\mathrm{opt} .}=\left[12+3 K_{\mathrm{D} 3}\left((1-R) / R^{2}\right)\right]^{-1}[4+[16 \\
& +12 K_{\mathrm{D} 0}-12 K_{\mathrm{D} 4}((1-R) / R) \\
& \quad+3 K_{\mathrm{D} 0} K_{\mathrm{D} 3}\left((1-R) / R^{3}\right) \\
& \left.\left.\quad-3 K_{\mathrm{D} 3} K_{\mathrm{D} 4}\left((1-R) / R^{4}\right)\right]^{1 / 2}\right] .
\end{aligned}
$$




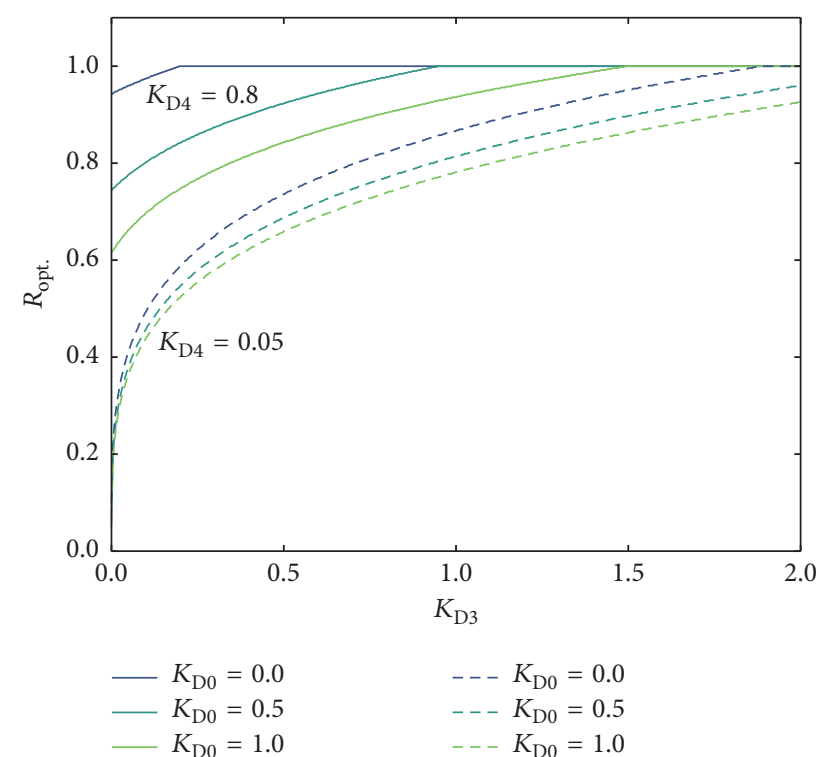

FIGURE 13: The optimum actuator size ratio $R_{\text {opt. }}$ (that will result in maximum hydraulic power density $\left.(1 / R) C_{\mathrm{P} \text { max. }} / C_{\mathrm{P} \text { ref. }}\right)$ plotted as a function of the two coefficients $K_{\mathrm{D} 3}$ and $K_{\mathrm{D} 4}$ for various values of the static drop coefficient $K_{\mathrm{D} 0}$.

The optimum duct size ratio given by this new model is plotted in Figure 13 (an expanded version of Figure 12) for a few arbitrary coefficient values.

The quality of the optimum predictions above depends on the validity of the starting hypotheses (which assume that the flow can be modeled as one-dimensional and that the flow regime around the duct remains constant). Nevertheless, those results, depending on two or three parameters only, are computed extremely rapidly. Importantly, insights over the key mechanisms relevant to the ducting of free-stream hydraulic machines are provided by the model-describing, in particular, why not all ducting is beneficial and how that is affected by the operating environment.

\section{Conclusions}

A one-dimensional model describing the fluid flow through hydraulic devices is a useful tool to characterize the performance of such machines operated in conditions where the mass flow rate is a control variable and the outlet water height cannot be controlled. This corresponds, for instance, to a small turbine or water wheel installed in a large river, or a machine operating in a cascading flow alongside a dam. In such a model, the device operating speed required to attain full load efficiency and the corresponding maximum hydraulic power can be quantified independently of the hydraulic efficiency.

In a machine of given frontal area operating at $100 \%$ load efficiency, it is shown that the use of a stationary duct permits an increase in actuator velocities but can only result in a reduction of the power coefficient. In specific cases, this may nevertheless result in an increase in power density (in addition to an expected change in the hydraulic and generator efficiencies).

Quantitative results can be provided using a simple model for evaluating pressure losses generated by a duct (with the implicit assumption that the duct flow regime is never changed). A single duct performance parameter $K_{\mathrm{D} 2}$ is then sufficient to determine numerically, for each installation, whether an optimal duct-to-actuator size ratio exists and to find its value. An illustrative model accounting for more subtle dependencies of the losses generated by ducting is also implemented. While such models are expected to require calibration and operating range restrictions in order to reflect results provided by experimental measurements, they provide design guidance at a computing cost many orders of magnitude smaller than those associated with CFD methods, which also require experimental validation to provide reliable results.

In future works, it may be possible to couple this framework with models describing the hydraulic and generator efficiencies of the machine, as well as to account for nonuniform inlet flow velocity distributions. Those extensions, coupled with numerical CFD simulations validated using water channel measurements, would lead to a powerful optimization tool to maximize the power generation of free-stream hydraulic devices.

\section{Nomenclature}

Symbols

$A_{\mathrm{A}}:$ Actuator frontal area $\left[\mathrm{m}^{2}\right]$

$A_{\mathrm{f}}$ : $\quad$ Device frontal area $\left[\mathrm{m}^{2}\right]$

$b$ : Width of rectangular channel [m]

$C_{\mathrm{P}}$ : Power coefficient (def. (8)) [-]

$e: \quad$ Specific mechanical energy $\left[\mathrm{J} \mathrm{kg}^{-1}\right]$

$F$ : $\quad$ Froude number [-]

$g:$ Gravitational acceleration $\left[\mathrm{m} \mathrm{s}^{-2}\right]$

$h$ : Height to water surface, positive upwards [m]

$H_{\text {eff. }}$ : Effective head (def. in Section 3) [m]

$K_{\mathrm{D}}$ : Drop coefficient (def. (16)) [-]

$K_{\mathrm{D} 0}$ : Static drop coefficient (def. (21)) [-]

$K_{\mathrm{D} 2}$ : Drag coefficient (def. (20)) [-]

$K_{\mathrm{D} 3}$ : Inner drag coefficient (def. (26)) [-]

$K_{\mathrm{D} 4}$ : Outer drag coefficient (def. (26)) [-]

$\dot{m}: \quad$ Mass flow $\left[\mathrm{kg} \mathrm{s}^{-1}\right]$

Q: $\quad$ Lanchester correction factor (cf. Section 4 and [16]) [-]

$q$ : Volume flow per unit width $\left[\mathrm{m}^{2} \mathrm{~s}^{-1}\right]$

$R: \quad$ Size ratio (def. (23)) [-]

$T$ : $\quad$ Generic parameter used for clarity (def. (18)) [-]

$u: \quad$ Velocity $\left[\mathrm{m} \mathrm{s}^{-1}\right]$

$\dot{\mathscr{V}}: \quad$ Volume flow rate $\left[\mathrm{m}^{3} \mathrm{~s}^{-1}\right]$

$\dot{W}$ : Mechanical power gained or lost by the fluid [W]

$z$ : $\quad$ Altitude, positive upwards [m]. 


\section{Greek Symbols}

$\alpha$ : Kinetic energy correction factor $[-]$

$\Delta:$ Net difference

$\eta$ : Efficiency [-]

$\rho$ : Density $\left[\mathrm{kg} \mathrm{m}^{-3}\right]$

\section{Subscripts}

$\begin{array}{ll}\text { A: } & \text { Actuator } \\ \text { alt.: } & \text { Due to altitude } \\ \text { av.: } & \text { Averaged in space } \\ \text { max.: } & \text { Maximum } \\ \text { opt.: } & \text { Optimum } \\ \text { loss: } & \text { Integral effect of pressure losses } \\ +: & \text { Immediately upstream of actuator surface } \\ -: & \text { Immediately downstream of actuator } \\ & \text { surface } \\ \infty: & \text { Far-field incoming conditions. }\end{array}$

Sign Conventions

Lengths: $\quad$ Lengths are measured positive upwards and downstream

Fluid properties: Fluid property changes are measured from the point of view of the fluid (i.e., negative values indicate a loss by the fluid, thus a gain for the machine operator).

\section{Conflicts of Interest}

The authors declare that there are no conflicts of interest regarding the submission of this manuscript.

\section{Acknowledgments}

The authors are grateful for the financial support provided by the Fluss-Strom project financed by the Bundesministerium für Bildung und Forschung (German Federal Ministry of Education and Research) under Project no. 1714.

\section{References}

[1] M. H. Mohamed, G. Janiga, E. Pap, and D. Thévenin, "Multiobjective optimization of the airfoil shape of Wells turbine used for wave energy conversion," Energy, vol. 36, no. 1, pp. 438-446, 2011.

[2] M. H. Mohamed, G. Janiga, E. Pap, and D. Thévenin, "Optimization of Savonius turbines using an obstacle shielding the returning blade," Renewable Energy, vol. 35, no. 11, pp. 2618-2626, 2010.

[3] L. Daróczy, G. Janiga, K. Petrasch, M. Webner, and D. Thévenin, "Comparative analysis of turbulence models for the aerodynamic simulation of H-Darrieus rotors," Energy, vol. 90, pp. 680-690, 2015.

[4] M. J. Khan, G. Bhuyan, M. T. Iqbal, and J. E. Quaicoe, "Hydrokinetic energy conversion systems and assessment of horizontal and vertical axis turbines for river and tidal applications: a technology status review," Applied Energy, vol. 86, no. 10, pp. 1823-1835, 2009.
[5] J. J. Cartelle Barros, M. Lara Coira, M. P. de la Cruz López, and A. del Caño Gochi, "Assessing the global sustainability of different electricity generation systems," Energy, vol. 89, pp. 473-489, 2015.

[6] Wachstumskern Fluss-Strom Plus, "Grundlastfähige Energiegewinnung durch ökologisch verträgliche Flusswasserkraftanlagen," German, October 2015, http://www.flussstrom.eu/.

[7] P. F. Pelz, "Upper limit for hydropower in an open-channel flow," Journal of Hydraulic Engineering, vol. 137, no. 11, pp. 1536-1542, 2011.

[8] M. Shives and C. Crawford, "Overall efficiency of ducted tidal current turbines," in Proceedings of the IEEE OCEANS 2010, pp. $1-6,2010$.

[9] T. Burton, N. Jenkins, D. Sharpe, and E. Bossanyi, Wind Energy Handbook, John Wiley and Sons, New York, NY, USA, 2011.

[10] Y. Li, "On the definition of the power coefficient of tidal current turbines and efficiency of tidal current turbine farms," Renewable Energy, vol. 68, pp. 868-875, 2014.

[11] M. Denny, "The efficiency of overshot and undershot waterwheels," European Journal of Physics, vol. 25, no. 2, pp. 193-202, 2004.

[12] E. Quaranta and R. Revelli, "Performance characteristics, power losses and mechanical power estimation for a breastshot water wheel," Energy, vol. 87, pp. 315-325, 2015.

[13] P. F. Pelz and M. Metzler, "An analytic approach to optimize tidal turbine fields," IOP Conference Series: Materials Science and Engineering, vol. 52, no. 5, Article ID 052019, 2013.

[14] M. Sánchez, R. Carballo, V. Ramos, and G. Iglesias, "Energy production from tidal currents in an estuary: a comparative study of floating and bottom-fixed turbines," Energy, vol. 77, pp. 802-811, 2014.

[15] G. Müller, S. Denchfield, R. Marth, and B. Shelmerdine, "Stream wheels for applications in shallow and deep water," in Proceedings of the Congress-International Association for Hydraulic Engineering \& Research, vol. 32, no. 2, p. 707, Venice, Italy, 2007.

[16] G. A. M. van Kuik, J. N. Sørensen, and V. L. Okulov, "Rotor theories by Professor Joukowsky: momentum theories," Progress in Aerospace Sciences, vol. 73, pp. 1-18, 2015.

[17] W.-H. Lam, L. Chen, and R. Hashim, "Analytical wake model of tidal current turbine," Energy, vol. 79, pp. 512-521, 2015.

[18] L. Chen and W.-H. Lam, "Slipstream between marine current turbine and seabed," Energy, vol. 68, pp. 801-810, 2014.

[19] G. T. Houlsby, S. Draper, and M. L. G. Oldfield, "Application of linear momentum actuator disc theory to open channel flow," Report OUEL 2296.08, 2008.

[20] K. H. Bergey, "The Lanchester-Betz limit (energy conversion efficiency factor for windmills)," Journal of Energy, vol. 31, no. 6, pp. 382-384, 1979.

[21] G. A. M. van Kuik, "The Lanchester-Betz-Joukowsky limit," Wind Energy, vol. 10, no. 3, pp. 289-291, 2007.

[22] F. W. Lanchester, "A contribution to the theory of propulsion and the screw propeller," Naval Engineers Journal, vol. 27, no. 2, pp. 509-510, 1915. 


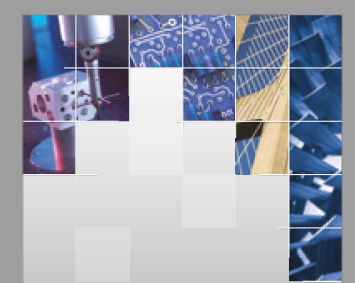

\section{Enfincering}
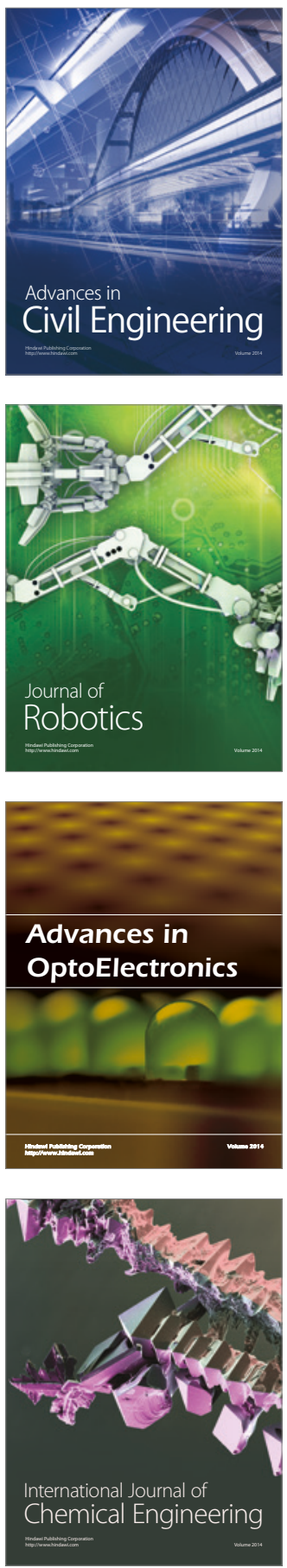

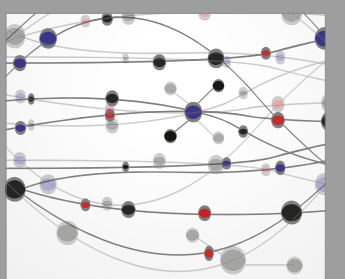

The Scientific World Journal

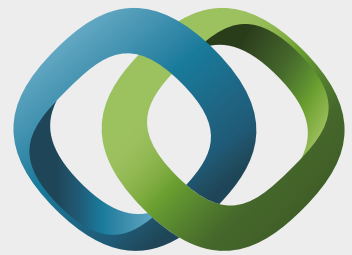

\section{Hindawi}

Submit your manuscripts at

https://www.hindawi.com
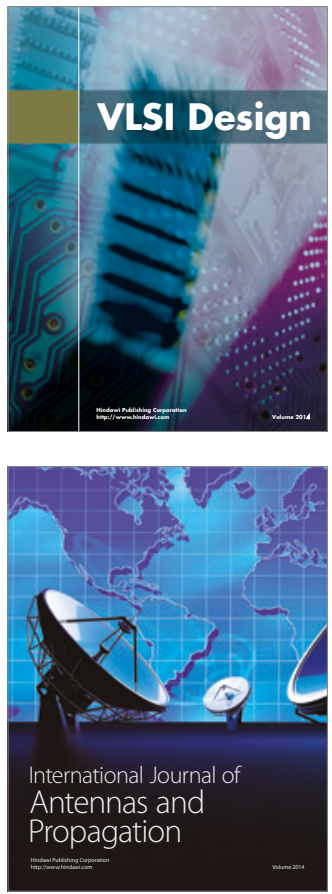

\section{Rotating}

Machinery
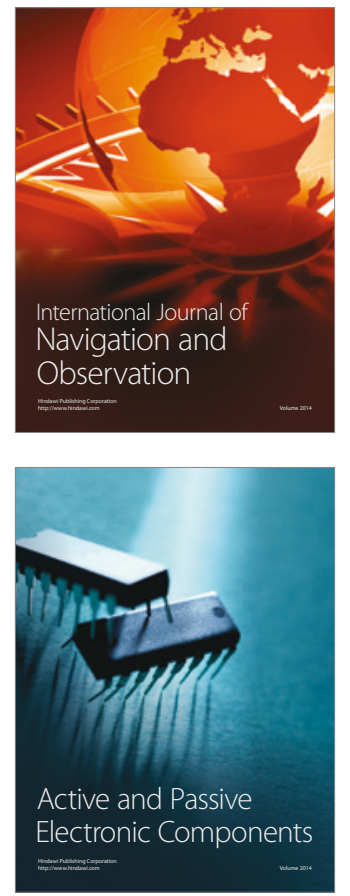
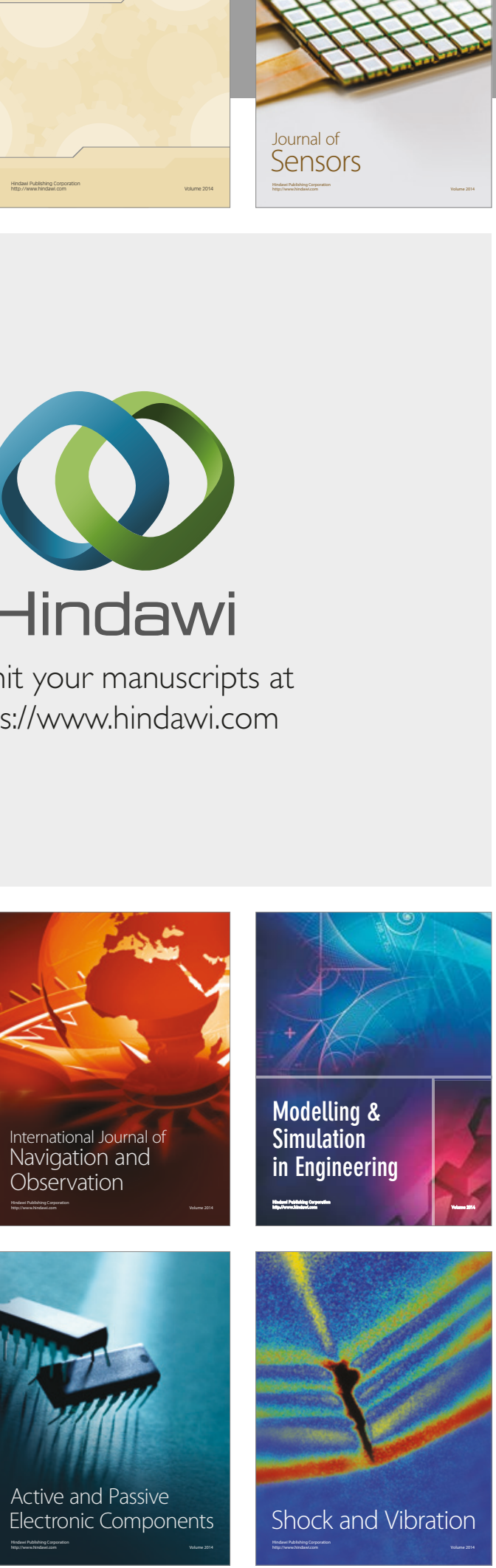
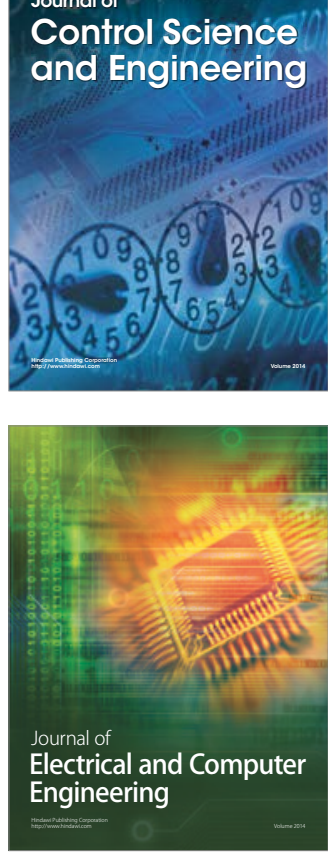

Distributed

Journal of

Control Science

and Engineering
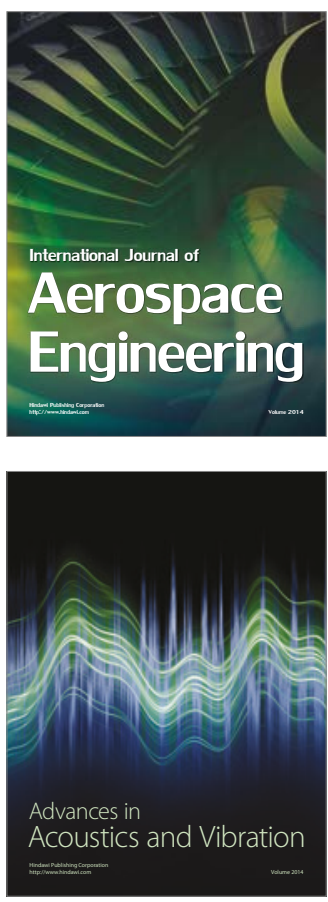

Sensor Networks 